\title{
A Study on Clinical Presentation of Dengue Fever in Children
}

\author{
Dr. Anjali S Raj ${ }^{1}$, Dr. Sucheta Munshi ${ }^{2}$, Dr. Bela H Shah ${ }^{3}$ \\ ${ }^{1}$ Resident in Department of Paediatrics, B.J.Medical College \& Civil hospital, Ahmedabad, India \\ ${ }^{2}$ Assistant Professor, Department of Paediatrics, B.J.Medical College \& Civil hospital, Ahmedabad, India \\ ${ }^{3}$ Professor and HOU, Department of Paediatrics, B.J.Medical College \& Civil hospital, Ahmedabad, India
}

\begin{abstract}
Dengue fever caused by dengue virus is not only versatile in its clinical presentation but also adds on to morbidity and mortality of human community. In this study we have made an attempt to describe the variety of clinical presentation of dengue fever, risk factors and complications of the illness in children. Incidence of dengue illness was 1.69\% of which 1-5 years was the most common age group affected with M:F ratio of 1.33:1. 89\% illness was seen in the post monsoon periods (August- November). Mean duration of fever in dengue illness was 5 days for DHF and DSS as compared to 4 days for dengue fever. Fever was most common presenting complaint followed by rash in $67 \%$ and vomiting\& abdominal pain $66 \% .88 \%$ had thrombocytopenia with mean platelet count was 66,070. Case fatality rate of $3.06 \%$ was encountered with severe refractory shock, DIC, ARDS, hepatic failure and neurological manifestations singly or in combination were the commonest causes of death.
\end{abstract}

Keywords: dengue fever, DHS,DSS, thrombocytopenia, refractory shock

\section{Introduction}

Dengue has emerged as a notable public health problem in recent decades in terms of mortality and morbidity associated with it.[1] It is one of the most important mosquito-borne viral disease affecting humans, occurring in over 100 countries and threatening the health of more than 2.5 billion people of the tropics and subtropics.

Dengue is endemic in many parts of India and epidemics are frequently reported from various areas within[2-4] and outside the country.[므, $\underline{6}] \mathrm{DF} / \mathrm{DHF} / \mathrm{DSS}$ are caused by four serotypes ( DEN 1-4) transmitted from viremic human beings to susceptible mainly by bites of AEDES AEGYPTI and AEDES ALPBOPICTUS mosquito species. ${ }^{[11]}$

The case fatality rate with dengue hemorrhagic fever (DHF) and dengue shock syndrome (DSS) can be as high as $44 \%$, seen mostly among children.[7] The majority of hospitalized children with dengue fever, DHF/DSS recover uneventfully with meticulous supportive treatment; however, a small percentage of critically unstable patients require paediatric intensive care unit (PICU) admission and can have significant mortality. Hence early and rapid laboratory diagnosis of dengue is crucial. Appropriate clinical management can save the lives of DHF and DSS patients and mortality can be reduced to less than $1 \%$.[ㅁ]

The aim of this present study was to review the classical as well as atypical clinical presentation of dengue illness along with its complications, co morbid conditions and outcome.

\section{Materials and Methods}

The authors examined the records of 196 consecutive patients with dengue infection admitted to paediatric ward of referral institute B. J. Medical College and Hospital Ahmedabad over a 2 year period beginning January 2010.
The charts were obtained from the medical records department and data was entered on a spread sheet by one of the authors and cross checked for accuracy and completeness by others.

Case Definition: During a prevailing epidemic, the clinical diagnosis of DHF is based on four major characteristic manifestations[1,]) ,a history of fever lasting for 2-7 days, hemorrhagic manifestations (petechiae, palatal or gum bleeds, hematemesis or malaena), thrombocytopenia (platelet count $<100,000$ cumm), evidence of plasma leakage manifested by hemoconcentration (an increase in the hematocrit $20 \%$ ) or pleural effusion or ascitis. [1,2 ]In children with DSS, the plasma leakage is extensive enough to result in shock. The diagnosis of dengue fever in the present study was based on clinical findings, but was confirmed by serology employing IgM and IgG-capture enzyme linked immunosorbent assay (MAC-ELISA) specific for dengue antibodies in patient's sera.

Disease severity was assessed according to the WHO grading system

Grade I - Positive tourniquet test

Grade II - Spontaneous bleeding

Grade III- Circulatory failure

Grade IV-Undetectable blood pressure and pulse

Grades III and IV DHF are also referred to as Dengue Shock Syndrome (DSS)

\section{Results}

Out of 11,560 admissions, 196 patients suffered from dengue illness making an incidence of $1.69 \%$. 96 (49\%) had dengue fever, $80(40.8 \%)$ had dengue haemorrhagic fever and $20(10.2 \%)$ had dengue shock syndrome. 1-5 years was the most common age group affected with M:F ratio of $1.33: 1$. In this study most of our patients $89 \%$ presented in the post monsoon periods (August- November). 


\section{International Journal of Science and Research (IJSR) \\ ISSN (Online): 2319-7064}

Index Copernicus Value (2013): 6.14 | Impact Factor (2015): 6.391

\section{Clinical Features}

- Varied degree of fever is seen in Dengue illness. It ranges from mild fever to high grade fever.

- Moderate fever was most commonly encountered seen in $106(54 \%)$ cases and hyperpyrexia was seen in 6(3\%) cases.

- Mean duration of fever in dengue illness was 5 days for DHF and DSS as compared to 4 days for dengue fever which can be correlated as DF if untreated can progress to DHF \& DSS. ${ }^{[21]}$

- Most common presentations were fever 196 (100\%), rash 132 (67\%), vomiting\& abdominal pain130 (66\%), cough/cold $82(42 \%)$ and myalgia63 (36\%) headache $68(34.6 \%)$, bleeding 62(31.6\%) breathlessness 32(16.3\%) convulsion / altered sensorium in $10(5.1 \%)$

- 2 patient of DF were having severe difficulty in walking due to dengue myositis, which was an atypical presentation not commonly observed in previous studies. $^{[21,22,23]}$

\section{Clinical signs}

Tachycardia was the most common clinical sign seen in this study with $72.4 \%$ incidence followed by congested face $101(51.5 \%)$, petechiae and purpura 99(50.5\%) ,hepatomegaly $92(46 \%)$, pedal edema $70(35 \%)$, pleural effusion $68(34.6 \%)$, ascites $56(28 \%)$, positive tourniquet test $32(20.7 \%)$, shock in $12(6.1 \%)$ and refractory shock was seen in $8(4 \%)$,drowsiness /stupor/coma was seen in 20 $(10.2 \%)$,jaundice in $8(4 \%)$ and bradycardia $7(3.5 \%)$.

\section{Haematological manifestations}

Of 196, 176 (89.7\%) had platelets < 1lakh, rest 20(10.3\%) had platelets above the mark.52 (26.5\%)had severe thrombocytopenia with platelets $<20,000$ of which 18 had disordered coagulation and 10 of them had DIC of which 6 expired who also had refractory shock. Mean platelet count was 66,070 .

Petechiae was the most common bleeding manifestation seen among 99(50.1\%) patient 's of which 95 had platelets counts $<50,000$.

GI bleeding was seen in $44(22 \%)$ all had platelets $<50,000$ of which 10 had massive GI bleeds of whom 3 remained in shock and also had DIC and died ultimately despite maximal supportive care and resuscitation.

In the present study 30(15.3\%)patients recovered their platelets within 48 hours of treatment.

\section{Hemodynamic and cardiovascular manifestations}

Shock was the most common and difficult to treat complications despite appropriate fluid management in accordance with WHO regimen. $20(10.2 \%)$ children had shock of which 7 were refractory to fluid therapy and blood products(whole blood, packed cell volume, FFP) given as indicated.

1 of 7 with persistent shock improved with ongoing supportive treatment, rest succumbed to death as they had additional complications like encephalopathy, ARDS and DIC.

\section{Abnormal fluid collection}

Apart from shock, third space loss of fluids was an important feature manifested as pleural effusion in 68 children (50.4\%). 56(46.7\%) had ascitis, 8 children had profound generalized anasarca and 2 children had pericardial fluid accumulation.

\section{Respiratory manifestations}

In addition to pleural effusion seen in $68(34.6 \%)$ patients, 8 satisfied criteria of ARDS of which 3 died . 11 patients needed ventilatory support, indications being ARDS in 6,3 cardiac arrest, 2 for neurological indications. 4 were weaned off ventilator with mean duration of need for ventilation being 2.3 days.

\section{Neurological manifestations}

20 patients presented with altered sensorium of which 12 had convulsions, 3 had status epilepticus, 5 of them remained in altered mental status underwent imaging with computerized tomographic (CT) scans and cerebrospinal fluid (CSF) analysis. The CSF was abnormal in three patients, who had raised protein and lymphocytic pleocytosis and CT scan of all of them had features of cerebral edema.

\section{Renal issues}

Acute renal failure was seen in 12 children who presented with oligouria or anuria with a mean creatinine of 3.4 $\mathrm{mg} / \mathrm{dl} .10$ of them improved by effective fluid management. 2 died of refractory shock.

\section{Co infections}

Malaria was found to be the most common co- infection associated with dengue infection. Of 196 patients, 4 had P.falciparum and 11 had P.vivax co-infection. 2 patients had mixed infection. 5 patients had enteric fever and 2 had hepatitis A infection.

\section{Duration of stay}

The mean duration of stay was 5.1 days for dengue fever, 7 days for DHF and 10 to 12 days for DSS

\section{Case fatality rate}

6 out if 196 cases died making a case fatality rate of 3.06\%.

\section{Cause of death}

- Refractory shock with DIC and ARDS - 3

- Refractory shock with DIC with encephalopathy - 2

- Refractory shock with encephalopathy - 1

\section{Discussion}

Here are reported variety in clinical presentation of dengue illness with special reference to its severe forms that required intensive management. The commonest indication of for PICU admission being persistent shock followed by respiratory distress and neurological symptoms .Findings not previously described in the setting of dengue illness included bone marrow suppression in 2 patients and myositis.

Although there was no sex predilection the illness occurred most often in age group $>5$ years, followed by 1-5 years which is different from previous studies, which has recorded 1-5 years to be the commonest one.

\section{Volume 5 Issue 4, April 2016}




\section{International Journal of Science and Research (IJSR) \\ ISSN (Online): 2319-7064}

Index Copernicus Value (2013): 6.14 | Impact Factor (2015): 6.391

With respect to circulatory complications, 7(3.5\%) presented with shock refractory to Emergency Room fluid resuscitation. An important cause of persistent shock in a patient with DSS whose circulatory status fails to improve despite adequate fluids is internal hemorrhage, hence all patients with fluid refractory shock received blood transfusion (packed red blood cells and plasma) unless hemoconcentration persisted.

A study of hemodynamic profiles in DHF from Thailand reported lowered cardiac index due to decreased ejection fraction and lowered preload 16 while reports from New Delhi, India have reported global

Hypokinesia [17,18]. Pericardial effusions have been reported previously, but did not contribute to the shock status[1]. Steroids were not used in cases of refractory shock as there was no data supporting it's use. Unsurprisingly, all four patients with uncorrected shock and DIC expired.

With respect to hematological complications, the mean hematocrit at presentation was $35.8+6.3 \%$.Indian children with DHF have a lower than expected rise in hematocrit during the plasma leakage period: this has been attributed to the high prevalence of iron deficiency anemia in the general population[21,22]. Platelet counts less than 50,000/cumm was noted in $65.3 \%$. DHF patients with a platelet count $<$ 50,000 /cumm have been reported to have a six-fold higher mortality than those with platelet counts $>50,000$ /cumm 24 and in the present study too, only two of nine deaths occurred in children with platelet counts greater than 50,000 /cumm. Immune related platelet damage and inhibition of platelet aggregation contribute to the thrombopathy[5].

Apart from decreased platelet number and function, DHF patients can have abnormal hemostasis (vasculopathy, coagulopathy and DIC [26,27].DIC and massive fatal bleeding may be more frequent in children with prolonged shock [27-29].

In the present study, while frank DIC was seen in 10 cases, co-existing refractory shock resulted in death in 4 children 11 children $(5.6 \%)$ required ventilation, the main indication being increasing respiratory distress. ARDS was seen in 6 children, 3 of whom died.

All cases of ARDS occurred in children presenting with Grade IV shock and lung injury in these patients may have resulted from "shock lung" and/or increased capillary permeability. B The need for high airway pressures required to ventilate patients with severe ARDS in DSS can easily further worsen the patients' already precarious hemodynamics and it is not surprising that Dengue associated ARDS is associated with a high mortality.

Hepatic and neurological dysfunction have been classified as "unusual complications/manifestations" of DHF. Hepatic dysfunction may be multifactorial - the most important causes are prolonged shock, associated metabolic acidosis and DIC with resultant ischemic hepatitis. The liver may also be the major site of dengue viral replication 6-9].
Only one-fifth of the 24 children with neurological manifestations were in shock. While children with DSS may have abnormal neurology secondary to cerebral hypoperfusion on account of shock leading to hypoxic ischemic events and/or intracranial bleeds, other significant reasons for neurological presentations include cerebral edema, direct neurotropic effect of dengue virus resulting in encephalitis /encephalopathy, or secondary to hepatic dysfunction and metabolic derangements such as hypoglycemia and hyponatremia.

Co-infections were seen in $12.2 \%$ and it is important that they be promptly recognized. Co-infections can modify the clinical presentation of dengue and result in missed or delayed diagnosis and treatment of dengue shock.

\section{Limitations}

The limitations of the present study relate to the inherent weakness of a retrospective analysis. The strengths relate to the fact that all cases were seen at a single centre by the authors where the indications for admission and subsequent management was standardized.

\section{Future Scope of the Study}

The clinical implications of our findings relate to the fact that critically unwell patients with dengue fever, DHF and DSS may have complications singly or in combination related to virtually every major system. Early recognition in conjunction with meticulous monitoring and targeted supportive care is the cornerstone of a successful outcome. So any patient with clinical features of dengue fever should be monitored intensively so as to prevent morbidity and mortality due to the disease.

\section{Conclusions}

Dengue fever is a common preventable vector borne disease common in both sexes with a predilection for higher age group. Fever with cough/cold, myalgia, arthralgia and weakness suggest milder form and altered sensorium, bleeding, suggest severe form of disease with thrombocytopenia being a consistent feature. A positive tourniquet test is a reliable early marker of severity Dengue can present atypically like dengue myositis, dengue hepatitis, bone marrow suppression and dengue encephalopathy. Severe refractory shock, DIC, ARDS, hepatic failure and neurological manifestations singly or in combination were the commonest causes of death in the present study.

\section{References}

[1] WHO report on global surveillance of epidemic prone infectious diease. (GAR) (www.who.int/csr/disease/dengue/en/ndex.htm/)

[2] WHO resources for prevention, control and outbreak response Dengue/DHF (www.who.int/csr/disease/dendue/dengueresources.pdf)

[3] WHO Dengue: guidelines for diagnosis, treatment, prevention and control New edition,2009 


\section{International Journal of Science and Research (IJSR) \\ ISSN (Online): 2319-7064}

Index Copernicus Value (2013): 6.14 | Impact Factor (2015): 6.391

(www.whqlibdoc.who.int/publication/2009/9789241548 71 eng.pdf)

[4] Guidelines for clinical management of dengue fever, DHF, DSS by National vector born disease control programme/Ministry of Health and Family welfare,2008(www.nvbdcp.gov.in)

[5] Scientific working group report on Dengue by TD/WHO 2006, (www.who.int/tdr)

[6] Situation update of dengue in the South East Asia region 2010(CDS Newsletter January 2011)(www.searo.who.in/Linkfiles/cdc_news_letter_vol 8issue_1pdf)

[7] $\overline{D H F}$ - early recognition/diagnosis/management, WHO 2006, WHO/Cbs/EPR/2006.4a

[8] Dengue hemorrhagic fever- diagnosis, treatment, prevention and control, WHO, geneva,1997.

[9] Guidelines for treatment of dengue/DHF in small hospitals, WHO, SEARO, NEW DELHI. 1999.

[10] WHO alert - prompt action needed on Dengue.by Dr. Samlee SEA/PR/1446, 09 august 2007.

[11] Nelson, Textbook of Pediatrics, $18^{\text {th }}$ edition.

[12] Harrison's Textbook of Internal Medicine, 18th edition.

[13]K Park, Textbook of Preventive and social medicine $18^{\text {th }}$ edition.

[14] Panikar, Ananthnarayan, Textbook of microbiology.

[15] IAP National Guidelines 2006.

[16] Guidelines for prevention and control of Dengue Fever/DHF, national institute of communicable disease, New

Delhi,2006(www.whoindia.org/linkfiles/CDGforPCDH F.pdf)

[17] Laboratory of infectious disase, National institute of infectious disease, Bethesda by Joseph E Blarney, Jennifer M Matro.(www.ncbi.nlm.gov)

[18] Role of platelet transfusion in management of Dengue in tertiary care centre(www.ajts.org) by R. K. Makroo, Apollo hospital, New Delhi.

[19] Carey L- Medin - Mechanism and role of viral protein in chemokine induction in Dengue fever.

[20] Observation on Hospitalised patientof Dengue fever. Study by carozon R Manoloto(www.escholarship.umassmed.edu_diss30)

[21] Manjinath Narayan et al. Study of children with Df in institute of social paediatrics, Govt Stanley Hospital, during Oct- Dec 2001, King's institute of preventive medicine. Chennai.

[22] Manjunath Kulkarni et al. clinco-epidemiological profile of children hospitalised with dengue, 2006. Jaipur.

[23] Hema Mittal et al : Clinicohematological Profile and Platelet Trends in Children with Dengue During 2010 Epidemic in North India

[24] Garg P. Utility of clinical improvement and platelet count recovery time in counseling children hospitalized with suspected dengue in a resource-poor setting. JCDR. 2008

[25] Tropical Medicine, Text book of infectious disease. 1963.

[26] Kapse AS Dengue illness IAP textbook of Pediatrics, 2nd edition 2002.

[27] Manual for Indoor Residual Spraying: Application of Residual Sprays for Vector Control
(http://www.paho.org/English/AD/DPC/CD/mal-whoinsecticide-manual.htm).

[28] Guidelines for Integrated Vector Management (http://www.afro.who.int/vbc/frameworkguidelines/guide_integrated_vector_management.pdf)

[29] Planning Social Mobilization and Communication for Dengue Fever Prevention and Control: A Step-by-Step Guide

(http://www.who.int/tdr/publications/publications/pdf/pla nning dengue.pdf)

[30]Dengue fever: new paradigms for a changing epidemiology Debarati Guha-Sapir and Barbara Schimmer.

[31] Internet references-

a) www.en.winkipedia/wiki/dengue-fever.

b) www.dhf.dde.moph.go.th/abstract/54.doc

c) www.cbroinfo.com/biological/pathogen/denv.htm.

d) www.ncbi.nlm.nin.gov/pubmed/9665979.

e) www.india.gov.in/sector/health family/vector born e01.php.

f) www.dhpe.org/infect/arbovirus.html.

g) www.nvbcdp.gov.in

[32] Sameer et al. Atypical manifestations of dengue by Sameer Gulati and Anu Maheshwari, Maulana Azad Medical College, New Delhi, India,2007

\section{Abbreviations}

DF Dengue fever

DHF Dengue hemorrhagic fever

DSS Dengue shock syndrome

WHO World health organisation

CSF Cerebro Spinal Fluid

ELISA Enzyme Linked Immunosorbent Assay

GIT Gastrointestinal tract

Hct Hematocrit

aPTT Activated Partial Thromboplastin Time

PT Prothrombin Time

FDD Fibrin Degradation Products

TT Torniquet Test

ORS Oral Rehydration Salt

PCM Paracetamol

OPD Outpatient Door

IVF Intravenous Fluid

Plt Platelet

BP Blood Pressure

CRT Capillary Refill Time

Max Maximum 